\title{
Reconfiguración de las dinámicas de la industria radiofónica colombiana en el ecosistema digital
}

\author{
Reconfiguration of the dynamics of the Colombian radio \\ industry in the digital ecosystem
}

\author{
Reconfiguração da dinâmica da indústria da rádio colombiana no \\ ecossistema digital
}

ANDRÉS BARRIOS-RUBIO, Universidad de Bogotá Jorge Tadeo Lozano, Bogotá, Colombia [andres.barrios@utadeo.edu.co] MARIA GUTIÉRREZ-GARCÍA, Universitat Autònoma de Barcelona, Barcelona, España (maria.gutierrez@uab.cat)

\begin{abstract}
RESUMEN
Las TIC reconfiguran la relación radio-audiencia, fenómeno en el que la industria establece rutas de acción y fija un campo de trabajo en la construcción permanente del entramado social. Este trabajo busca comprender y determinar la preparación que tiene el medio para afrontar los retos del entorno digital de las audiencias, mediante una metodología que combina herramientas cuantitativas (seguimiento de sus acciones en antena, redes sociales, web-r@dio) y cualitativas (entrevista semiestructurada, análisis de los mensajes y la composición de la web-r@dio). Los resultados evidencian una estrategia integrada de antena y pantalla, en $360^{\circ}$, para captar la atención del oyente-usuario.
\end{abstract}

Palabras clave: web-r@adio; radio; audiencia; modelo de negocio; rutina productiva.

\section{ABSTRACT}

ICTs reconfigure the radio-audience relationship, a phenomenon in which the industry establishes routes of action and sets a field of work in the permanent construction of the social fabric. This work seeks to understand and determine the preparation of the media to meet the challenges of the digital environment of the audience, through a methodology that combines quantitative (monitoring of their actions on the air, social networks,web-r@dio) and qualitative tools (semi-structured interview, analysis of the messages and the composition of the web-r@dio).The results show an integrated antenna and screen strategy, in $360^{\circ}$, to capture the attention of the listener-user.

Keywords:web-r@adio; radio; audience; business model; productive routine.

\section{RESUMO}

As TIC reconfiguram a relação rádioaudiência, um fenômeno em que a indústria estabelece rotas de ação e estabelece um campo de trabalho na construção permanente do tecido social. Com uma metodologia que combina ferramentas quantitativas (monitoramento das ações no ar, redes sociais,web-r@dio) e qualitativa (entrevista semi-estruturada, análise das mensagens e composição daweb-r@dio), este trabalho visa compreender $\mathrm{e}$ determinar a preparação do meio para enfrentar os desafios colocados pelo ambiente digital do público. Os resultados mostram uma estratégia de tela e antena integrada, em $360^{\circ}$, para capturar a atenção do usuário ouvinte.

Palavras-chave:web-r@adio; rádio; público; modelo de negócios; rotina produtiva.

Forma de citar:

Barrios-Rubio, A. \& Gutiérrez-García, M. [2017). Reconfiguración de las dinámicas de la industria radiofónica colombiana en el ecosistema digital. Cuadernos.info, [41], 227-243. https://doi.org/10.7764/cdi.41.1146 


\section{INTRODUCCIÓN}

La convergencia digital, la apropiación y el uso de narrativas transradiofónicas para llegar a las audiencias a través de los dispositivos de pantalla, así como la gestión de la información, suponen una transformación del ecosistema comunicativo de la industria radial que exige comprender y redefinir el medio, los contenidos y la profesión. Es un proceso de construcción de sentido social, de creación de imaginarios, producción y circulación de contenidos, sostenido por un esquema convergente de actuación que alude a la integración de modos de comunicación textual, sonora, visual e iconográfica que conducen hacia la cross-radio.

En este contexto, la elaboración y difusión de contenidos radiofónicos responde a nuevas dinámicas espacio temporales de consumo que, por una parte, rompen la linealidad y unidireccionalidad de la comunicación radio-audiencia y, por otra, implican una reconfiguración de las rutinas productivas y del modelo de negocio. Aunque para la industria radiofónica colombiana el sonido sigue siendo su core businnes, las emisoras y cadenas se reinventan para adaptarse a la convergencia y a las plataformas, apostando, eso sí, por nuevos formatos y formas de captar la atención del público. Estos recursos narrativos y mediaciones técnicocomunicativas reconceptualizan los patrones de actuación del medio radiofónico y su papel en la sociedad colombiana. Como el mercado radiofónico colombiano responde a unos requerimientos propios, se hace necesario construir una mirada que analice y valore su proceso de reconfiguración de lo analógico a lo digital.

\section{MARCO TEÓRICO}

El proceso de migración y convergencia de contenidos radiofónicos en la red busca crear una asociación de carácter simbiótico, generando un nuevo modelo de actuación basado en la confluencia de recursos expresivos y procesos interactivos (Sosa, 2000). Esta evolución convierte a la radio convencional en un cibermedio; esto, según Cebrián (2009), implica el desarrollo - por parte del productor-emisor de contenidos-de estrategias adecuadas a la plataforma de Internet que integren diferentes sistemas expresivos para llegar a usuarios con los que puede dialogar e, incluso, intercambiar roles. Desde esta perspectiva, el funcionamiento del medio en el ecosistema digital ha cambiado la forma de concebir la comunicación radiofónica (González \& Salgado, 2011), cuyo epicentro ya no se concentra exclusivamente en la antena, sino también en la creación de sinergias que concurren en una plataforma web que debe responder a las nuevas dinámicas de consumo (Amoedo \& Martínez-Costa, 2016) de una audiencia inmersa en el ecosistema digital (Peñafiel, 2016; Cerezo, 2016; Meso, Larondo, Peña, \& Rivero, 2014; Gutiérrez, Monclús, \& Martí, 2014; López, 2005). Este está regido por dispositivos de pantalla (Fernández, 2014) y por una ruptura del esquema de consumo en tiempo lineal que implica, además, la creación de contenidos sonoros que serán distribuidos por la industria desde sus portales y plataformas digitales (Martínez-Costa, 2015). Este conjunto de factores comporta la evolución conceptual y estratégica de la ciberradio a la webr@dio que se convierte en el núcleo de las operaciones comunicativas de la industria y en el espacio desde el que se distribuyen contenidos en streaming, a la carta, como podcasts, y se estimulan las dinámicas de interacción con la audiencia, ya sea a través de redes sociales o foros (Barrios \& Gutiérrez, 2016b).

La radio busca consolidarse como un medio líder, tanto en su formato convencional como digital, a nivel nacional e internacional, centrando sus rutinas productivas entorno a la información, la música, el entretenimiento y el conocimiento. Por esa razón, suele presentar estrategias administrativas que responden a las necesidades, expectativas y requerimientos de los stakeholders de la emisora. Esto ocurre en el contexto de convergencia de escenarios que conlleva la reestructuración de la empresa informativa (Campos-Freire, 2015; García-Alonso, 2014; Preciado \& Monsalve, 2008; Batista, 2004) y encuentra su éxito en la explosión de seguidores digitales que se logra captar, trabajo bajo un sistema 24/7 (Piñero-Otero \& Videla, 2013; Salaverría, 2010; Rivadeneyra, 2008) que produce contenidos para un uso y canal de distribución determinado (Martínez-Costa, 2015).

Las dinámicas del mercado proponen un replanteamiento del medio y sus estrategias, ya que "la producción, circulación y apropiación de los productos comunicativos tienen como base los cuatro pilares de la sociedad del conocimiento: acceso e información para todos, libertad de expresión y diversidad lingüística" (Barrios, 2016, p. 134). Así, la web-r@dio, epicentro de la acción, incide en los profesionales y en sus rutinas productivas, en las audiencias y en los procesos de distribución y consumo de productos (Barrios \& Zambrano, 2015; Barrios, 2015; Arrojo, 2015; García-de-Torres, 2010).

Desde la industria radiofónica, sumida en un proceso de convergencia (Soengas, 2013; Ortiz \& López, 
2011; Salaverría \& García, 2008; Domingo et. al., 2007; Pavlik, 2004), se diseñan tácticas para afrontar la desafección juvenil (Barrios \& Gutiérrez, 2016a; López \& Gómez, 2014; Gutiérrez et al., 2014) y responder a los nuevos hábitos de consumo en un contexto altamente competitivo (Yuste, 2015; López \& Martínez, 2014; Perona, Barbeito, \& Fajula, 2014), en el que gracias al smartphone-el ciudadano desarrolla un sinnúmero de actividades como acceder a los contenidos radiofónicos mediante aplicaciones (Piñero-Otero \& Videla, 2013). En este punto, es interesante destacar el énfasis de la industria por atender a las necesidades y expectativas de los usuarios (Ribes, Monclús, Gutiérrez-García, \& Martí, 2017; Bonini \& Monclús, 2015; Huertas \& Figueras, 2014; Ayala, 2014).

En el entorno digital, la verticalidad del esquema comunicativo radiofónico se diluye buscando una horizontalidad que las redes sociales propician. Desde esta óptica, se potencia la conversación del medio con los seguidores y de estos entre sí (Ramos del Cano, 2014; Scolari, 2013; Cebrián, 2009; Sosa, 2000). Así, la industria radiofónica comprende que la audiencia tiene unas competencias distintas y se rige por unas coordenadas espacio-temporales en las que busca el contexto de las noticias y los significados e implicaciones para el entramado social. El público de hoy es tornativo, iconográfico, con inteligencia social y emocional colectiva (Barrios, 2016).

Las rutinas productivas de la radio están envueltas en etiquetas y códigos que recopilan información; así, el medio se empodera, expande sus horizontes y el servicio cambia (Bardoel, 2007). El tema expuesto tiene muchas aristas en el campo de la investigación en comunicación en el contexto colombiano, donde la radio - pese a ser uno de los medios con mayor penetración-ha sido poco estudiada a lo largo de la historia por parte de los científicos nacionales. En un momento de convergencia y transición como el que atraviesa el medio, es importante centrar la mirada en esa transformación de la industria radiofónica, consecuencia del proceso de digitalización, en la distribución y recepción de contenidos producidos desde el medio convencional.

\section{METODOLOGÍA}

La industria radiofónica colombiana ${ }^{1}$ y sus agentes comienzan a comprender las necesidades de un público que está inmerso en el entorno digital, usuarios ávidos de nuevas propuestas y oportunidades comunicativas. El objetivo de este texto es abordar la radio colombiana y sus dinámicas para afrontar los retos planteados por el entorno digital de las audiencias. Para focalizar el eje de desarrollo del trabajo de estudio de caso (Martínez, 2006; Arzaluz, 2005; Yacuzzi, 2005; Stake, 1998) se plantearon dos preguntas de investigación, desde las que se articula el análisis del fenómeno:

P1. ¿Cuál es el impacto de las TIC en el quehacer y en la relación con la audiencia de un medio de comunicación como la radio?

P2. ¿Cómo es la interconexión antena-ecosistema digital en la emisión diaria de la radio?

Para la selección de la muestra se recurrió a los datos del Estudio Continuo de Audiencia Radial (ECAR) 2016-3, realizado por el Centro Nacional de Consultoría (2016), que revela:

1. La fuerte penetración de la radio comercial (97\%) frente a las propuestas de interés público $(2 \%)$ y comunitarias (1\%) y

2. El dominio del mercado generalista por parte de dos cadenas (Caracol -53\%-y RCN -24\%-) y la emisora Blu Radio (20\%).

Los criterios expuestos suponen obviar las alternativas de interés público y comunitarias dada su baja incidencia en el mercado y centrar la mirada en las emisoras generalistas, dado que la ideología e inversión de la industria está concentrada en ellas (Barrios, 2016). Además, según ECAR 2016-3, las cinco estaciones radiofónicas con mayor número de oyentes (tabla 1) son Caracol Radio y W Radio de la cadena Caracol, RCN Radio y La FM de la cadena RCN y Blu Radio, una estación que nació en 2012 y que en cuatro años se ha posicionado en el segundo puesto del ranking de audiencia.

Una vez seleccionadas las emisoras, y para dar respuesta a las preguntas de investigación, se diseñó un instrumento metodológico cualitativo -entrevista semiestructurada (Ardèvol, Bertrán, Callén, \& Pérez, 2003) y de análisis de contenido de las emisoras en antena (Colussi, 2013; Martí, 2011)-y uno cuantitativo -seguimiento de la actividad del emisor en las redes sociales y las web-r@dio (Barrios, 2013)-. Las entrevistas se realizaron a directivos y agentes del medio radiofónico (tabla 2), referentes de dichas emisoras, para establecer cómo se aborda el fenómeno tecnológico en 


\begin{tabular}{lcc}
\hline Caracol & Caracol Radio & 1.749 .000 \\
\cline { 2 - 3 } & W Radio & 1.089 .500 \\
\hline RCN & RCN Radio & 648.100 \\
\hline Blu Radio & La FM & 649.000 \\
\hline
\end{tabular}

Tabla 1. Sintonía de las principales emisoras generalistas de Colombia

Fuente: Elaboración propia con los datos ECAR 2016 (tercera ola).

\begin{tabular}{|c|c|c|}
\hline $\begin{array}{l}\text { No. de } \\
\text { entrevistas }\end{array}$ & Cargo & Descripción \\
\hline 3 & Presidente & $\begin{array}{c}\text { Máxima instancia administrativa que } \\
\text { determina objetivos estratégicos a los que } \\
\text { apuesta la estación radial. }\end{array}$ \\
\hline 5 & Director de departamento & $\begin{array}{l}\text { Gestor táctico que delinea las acciones a } \\
\text { seguir en antena y el ecosistema digital para } \\
\text { captar la atención de los usuarios con su } \\
\text { equipo de trabajo online y offline. }\end{array}$ \\
\hline 10 & Integrante de programa & $\begin{array}{c}\text { Agente encargado de la producción y } \\
\text { realización del producto comunicativo sonoro } \\
\text { que sale al aire, en antena, y es llevado por el } \\
\text { equipo digital a la red. }\end{array}$ \\
\hline
\end{tabular}

Tabla 2. Agentes radiofónicos entrevistados y su función

Fuente: Elaboración propia.

cada una de las cadenas, la influencia de las redes sociales en el quehacer de la radio, los desafíos del entorno digital e identificar los valores que rodean esa comunicación medio-usuario.

Para efectuar la recolección del corpus sonoro de las emisoras, objeto de estudio, se recurrió nuevamente al ECAR 2016-3, que constata una significativa concentración de oyentes en la franja prime time (Caracol Básica-6am, Hoy por Hoy 937.100, La W-W Radio 884.200, RCN Básica-RCN Noticias 381.800, La FMNoticiero de la FM 314.400, y Blu Radio-Mañanas Blu 590.300). En su conjunto, la muestra se compone de 100 horas de audio (20 por cada emisora), 8089 tuits y 1048 post procedentes de los perfiles de las emisoras en las redes sociales y 25 capturas de pantalla de las web-r@dio (1 diaria por cada estación), bajo la estrategia metodológica de semana compuesta (Barrios \& Gutiérrez, 2016b) durante el mes de noviembre de 2016.
El objetivo era tener un cruce de variables (características del medio radial en su actuación en redes sociales, características del contenido que circula en la red y estrategias que se emplean en la interacción con los usuarios) para establecer la caracterización del comportamiento del medio (Bernal, 2006).

\section{RESULTADOS}

CONCEPCIÓN DEL NUEVO PANORAMA POR PARTE DE LOS AGENTES RADIOFÓNICOS

El análisis de las entrevistas (figura 1) muestra la concepción de un fenómeno tecnológico en permanente resignificación y reinvención para comunicarse e interactuar desde la particularidad de las micro-redes que se establecen en los perfiles de las plataformas de comunicación. Desde el discurso de los agentes de la industria radiofónica, se hace evidente que el contenido 


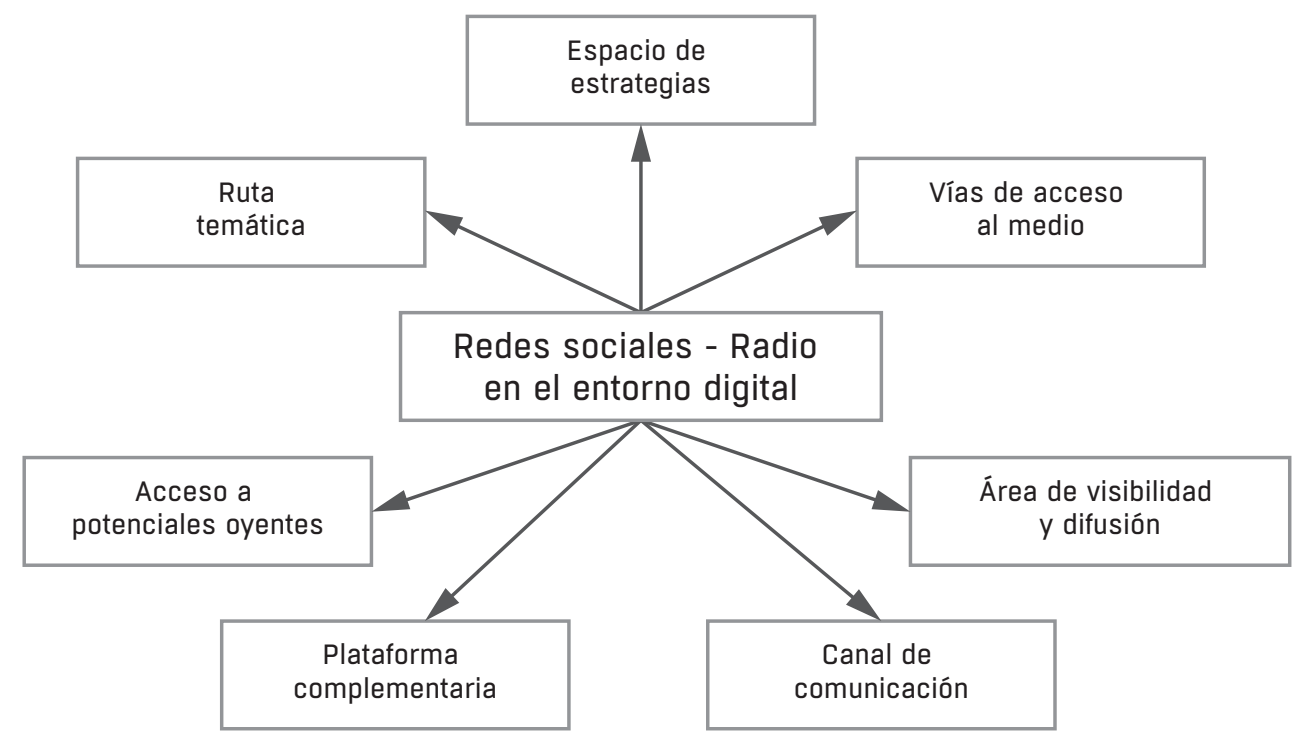

Figura 1. Categorización de lo manifestado por los actores de radio en el componente redes sociales - radio en el entorno digital

Fuente: Elaboración propia.

pasó a un segundo plano y que lo que realmente importa es la estrategia de contacto y de participación en un escenario de relación entre el medio y los sujetos, un fenómeno de socialización y reconocimiento.

Los agentes radiofónicos (figura 1) evidencian una preocupación latente por lograr el mayor número de seguidores posibles en las cuentas corporativas de los medios. Desde las redes sociales, se establecen tácticas para impactar en el consumo de productos comunicativos por parte de los usuarios en el entorno digital. Se apuesta por una bifurcación convergente de emisión y distribución que posiciona la marca de la emisora en el mercado, ajustada a la diversidad de escenarios a los que accede el usuario desde su PC y dispositivos portátiles. Se apuesta por un seguidor endogámico, que no desvía su atención y termina reafirmando aquello que piensa y cree:

La radio hablada tiene que ser una radio muy influyente, independiente, transgresora, una alternativa, no lo mismo de siempre (...). Nosotros somos una emisora más joven que la competencia, y por eso somos más transgresores, apostamos por proyectar una vena digital que dinamice el producto en antena, la web, las redes sociales y las apps. Hay que innovar y ver el medio de otra manera, ser muy influyentes (Vicepresidente de radio - Blu Radio).
La industria radiofónica transita por el lenguaje hipermediático, que busca la fórmula idónea para conjugar la creación y la producción de contenidos que logren interesar, informar y entretener a una audiencia heterogénea. Se estructura un lenguaje descriptivo y coloquial, que conduce a unas acciones de consumo determinadas por parte del emisor. En la antena y en el ecosistema digital, se tiende a mantener una unidad conceptual en la que la comunicación radial y la actividad periodística se llevan a cabo en condiciones diferentes a las que permitía el medio convencional:

Ahora nuestro trabajo exige tener un pensamiento global, pero unas estrategias de acción locales (...). La globalización de los medios nos da una visión de mundo, pero como productores de contenido nos lleva a ser muy locales en el desarrollo de los mismos, de manera tal que nos conectamos con ese oyente y usuario de nuestra propuesta comunicativa (Director de programa-RCN)

Desde el proceso de reinvención y reconfiguración del modelo de negocio (figura 1), la industria radiofónica colombiana ofrece nuevas alternativas que permiten al oyente soportar el cambio de lo analógico a lo digital con un sonido más limpio, una cobertura sin interferencias y sin problemas territoriales, una variedad 


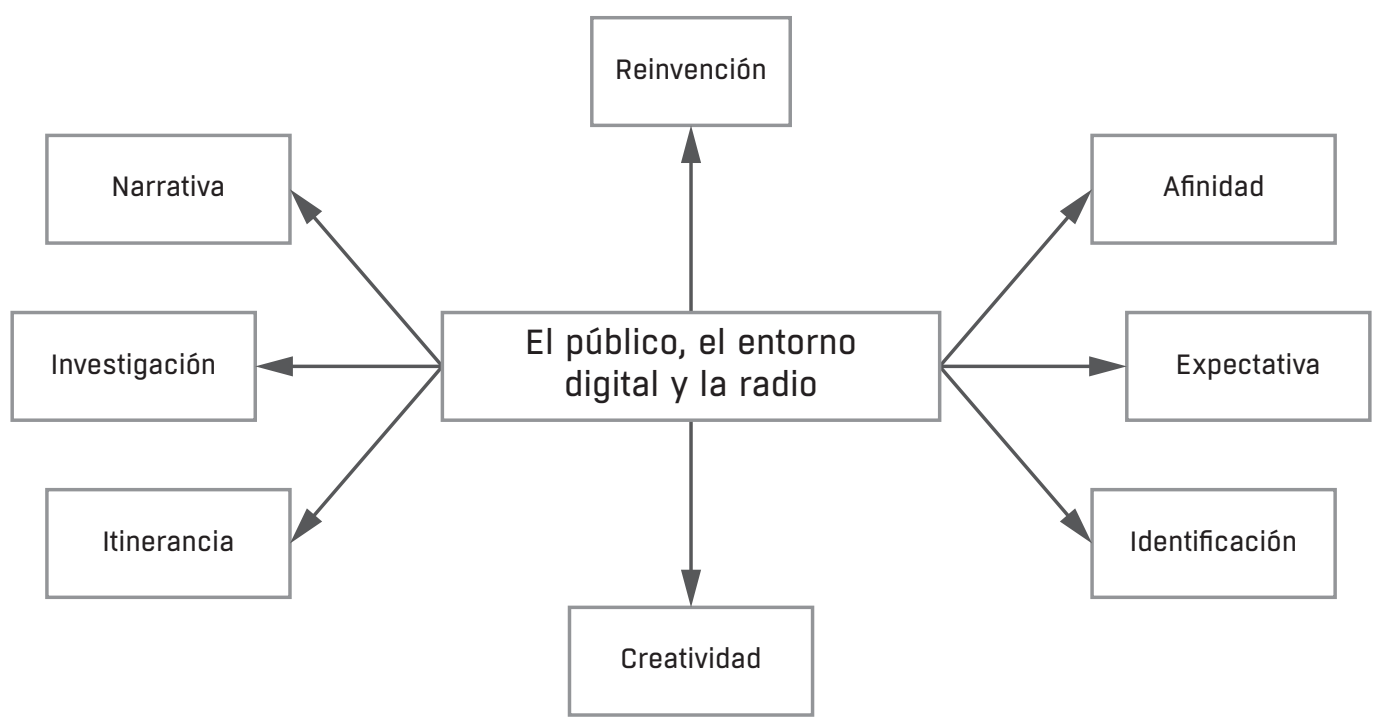

Figura 2. Categorización de lo manifestado por los actores de radio en el componente el público, el entorno digital y la radio

Fuente: Elaboración propia.

más amplia de canales, y una nueva opción de complementar el contenido que se ofrece en antena. Esta transformación tiene su eje central en el acceso, generación, procesamiento y transmisión de información:

Nuestra tarea hoy es fundamentalmente la de un chef de cocina, que tiene que satisfacer a todos los comen-

sales, (...) con contenidos amenos, livianos, musicales, culturales, deportivos, agradables, y por eso los equipos de trabajo están conformados por especialistas. Adultos con experiencia y jóvenes que responden a las necesidades de consumo en cada uno de los escenarios en los que está nuestra marca radiofónica (Director de departamento - Caracol Radio).

Para los agentes entrevistados, si bien los medios crean su audiencia bajo unas características y principios acorde a su estrategia empresarial (figura 2), hoy existe una complicidad entre el emisor y el receptor bajo un panorama en el que los dos son protagonistas del contenido que circula por las ondas hertzianas. Las audiencias, como productoras de información, comunicación y contenidos, guían al periodista en aquello que es de interés común y que, de una u otra manera, se vuelve tendencia no solo del medio, sino también de las redes sociales. Esta circunstancia afecta a los tradicionales roles profesionales dado que se asume la función de gestor de contenidos y estratega para dar respuesta a esos comentarios que emanan de los usuarios.
La propuesta comunicativa de la industria radiofónica presenta una estrategia triangular que gira en torno a tres pilares: los objetivos comunicativos del medio, las necesidades del mercado y la interacción de los usuarios en el ecosistema digital (figura 2). Se trata de un ejercicio básico que migra características del periodismo tradicional a la construcción del producto digital respondiendo a unos cuestionamientos básicos: ¿Qué se quiere lograr? ¿Qué se quiere producir, circular y hacer consumible? ¿Qué papel quiere jugar el medio frente a las redes sociales por las que van a circular contenidos? Las tecnologías de comunicación mediáticas y mass-mediáticas están modificando las metodologías de análisis e, incluso, sus marcos conceptuales bajo dos coordenadas que la definen: la expansión del relato a través de varios medios y la colaboración de los usuarios en ese proceso expansivo. La construcción de las dinámicas de acción del medio continúa concentrada en la antena, aunque se reconceptualiza para incorporarse al ecosistema digital (tabla 3).

Lasweb-r@dio determinan unos valores de relación entre el medio y sus usuarios (figura 3). Se trata de mecanismos ligados a la arquitectura de la información y la sindicalización de los contenidos que implican tácticas de distribución de información alineadas con la propuesta sonora de la estación 


\begin{tabular}{|c|c|}
\hline \multirow[t]{5}{*}{ Los medios en el entorno digital } & Intermediación e interactividad \\
\hline & Propagación de conocimiento \\
\hline & Identidad digital \\
\hline & Captación de nuevos públicos \\
\hline & Accesibilidad y uso por parte de los usuarios \\
\hline \multirow{4}{*}{$\begin{array}{l}\text { Las redes sociales y } \\
\text { las nuevas tecnologías } \\
\text { en el entorno de los medios }\end{array}$} & Conectividad constante a través de diversas plataformas \\
\hline & Competencia constante entre portales web y medios tradicionales \\
\hline & $\begin{array}{l}\text { Contenidos con criterios y estándares del periodismo (responsabilidad social, } \\
\text { manejo de fuentes, investigación, seguimiento, contextualización y desarrollo) }\end{array}$ \\
\hline & Dar voz al receptor \\
\hline \multirow{5}{*}{$\begin{array}{l}\text { La radio en el marco de la } \\
\text { sociedad digital }\end{array}$} & Reinvención e integración al entorno multimedia \\
\hline & Propuestas independientes y alternativas para un público más amplio \\
\hline & Nutrirse de las redes sociales \\
\hline & Seguimiento a los receptores \\
\hline & Construcción de contenido \\
\hline \multirow{6}{*}{$\begin{array}{l}\text { Los medios y sus periodistas } \\
\text { presentes en las redes }\end{array}$} & Precisar el objetivo de estar presente en las redes sociales \\
\hline & Sintetizar \\
\hline & Centrarse en la acción \\
\hline & Fijar objetivos para que se pueda llegar a más público \\
\hline & Consolidar la marca \\
\hline & Crear un canal de comunicación bidireccional entre emisor y receptor \\
\hline
\end{tabular}

Tabla 3. Características que debe incorporar la radio en el campo digital

Fuente: Elaboración propia.

radiofónica convencional. El estar presentes en el entorno digital demanda a los agentes del medio no solo producir contenidos totalmente nuevos, sino también enfrentarse a la emergencia de nuevos roles profesionales, además de una inevitable transformación de su modelo de negocio. Asimismo, las industrias locales se convierten en proveedoras de información y entretenimiento.

\section{LA RADIO EN EL ENTRAMADO DE LAS REDES SOCIALES}

Los medios tradicionales a los que se hace referencia en este estudio han alcanzado un importante número de amigos y seguidores en las redes sociales (tabla 4), siguiendo así la tendencia de la antena (ECAR 2016-3). Estos datos evidencian que la marca busca generar presencia digital y establecer un punto de encuentro con su audiencia. 


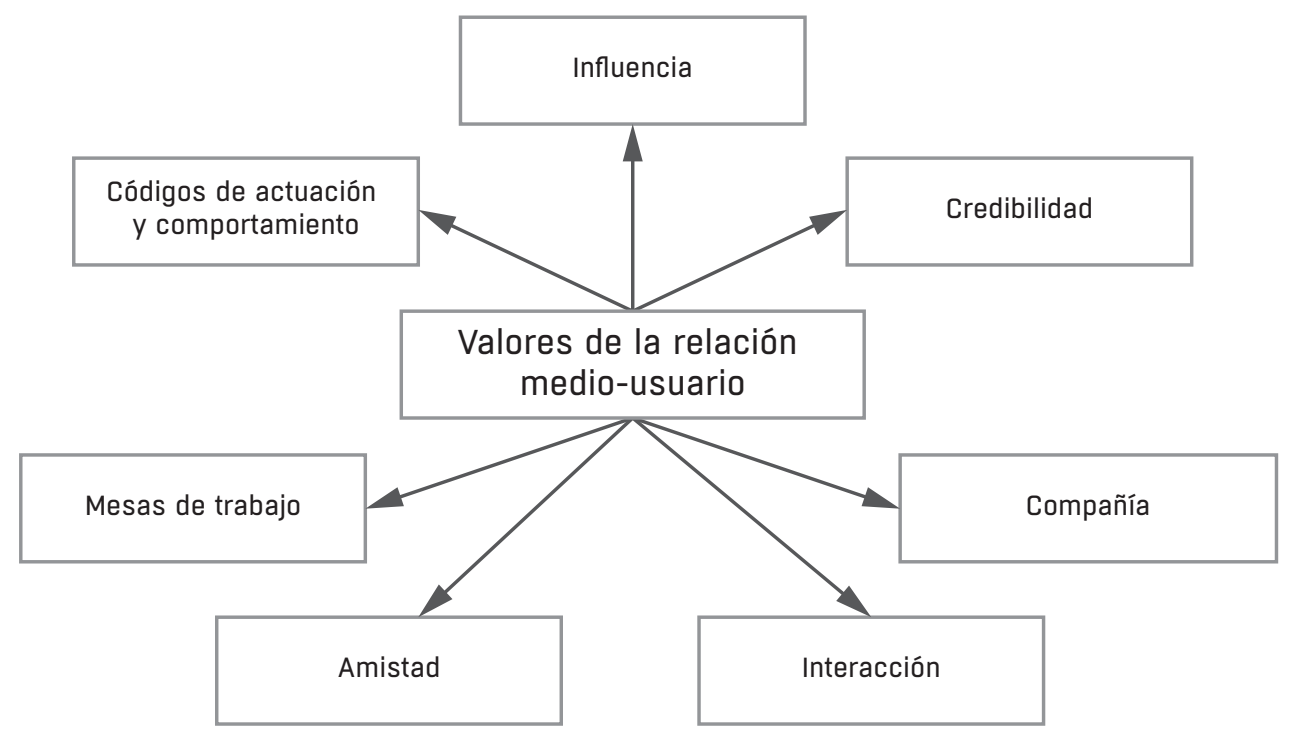

Figura 3: Categorización de lo manifestado por los actores de radio en el componente valores de la relación medio-usuario

Fuente: Elaboración propia.

Seguidores en Twitter

Seguidores en Facebook

\begin{tabular}{rll}
\hline Caracol Radio & 2.941 .875 & 580.039 \\
\hline W Radio & 2.969 .949 & 213.759 \\
\hline RCN Radio & 2.320 .610 & 366.382 \\
\hline La FM & 1.685 .766 & 659.241 \\
\hline Blu Radio & 1.842 .155 & 832.949 \\
\hline
\end{tabular}

Tabla 4. Seguidores de las emisoras en las redes sociales (noviembre de 2016)

Fuente: Elaboración propia.

El análisis de las cuentas muestra un uso continuado y equilibrado a lo largo de la jornada por parte del emisor, es decir, no existe una franja horaria con mayor flujo de publicaciones. Ahora bien, esta estrategia no siempre es garantía de éxito (tabla 5), pues los RT y Favoritos que se consiguen difieren en las horas del día, una circunstancia más ligada a las actividades del usuario en las redes sociales que a los perfiles de las emisoras. Sin embargo, se aprecia un esfuerzo por convertirse en tendencia en la red a través de los hashtags, una estrategia que apuesta por dar visibilidad a la marca y dotar de credibilidad a los contenidos.
Las constantes publicaciones del emisor generan dinámicas de respuestas de los usuarios, demostrando que la industria radiofónica generalista tradicional continúa siendo un referente informativo en el entorno digital. De hecho, los jóvenes más proclives a la escucha de la radio musical recurren a las estaciones matrices de las casas radiales en la red para aproximarse al entramado social del país.

Pese a que el número de amigos en Facebook no es significativo, se puede hablar de una relación de amistad y afinidad dado el alto número de comentarios que realizan los usuarios. Quizás la razón sea el interés por 


\begin{tabular}{|c|c|c|c|c|c|}
\hline & Caracol Radio & W Radio & RCN Básica & La FM & Blu Radio \\
\hline Tuits analizados & 1.595 & 1.091 & 1.086 & 1.783 & 2.007 \\
\hline $\begin{array}{l}\% \text { tuits con } \\
\text { hipervínculos }\end{array}$ & $81 \%$ & $99 \%$ & $34 \%$ & $86 \%$ & $57 \%$ \\
\hline $\begin{array}{l}\% \text { tuits con } \\
\text { hashtags }\end{array}$ & $59 \%$ & $40 \%$ & $61 \%$ & $100 \%$ & $91 \%$ \\
\hline $\begin{array}{l}\% \text { de } \mathrm{RT} \text { que } \\
\text { recibe }\end{array}$ & $69 \%$ & $87 \%$ & $74 \%$ & $69 \%$ & $61 \%$ \\
\hline $\begin{array}{l}\% \text { de Favoritos } \\
\text { que recibe }\end{array}$ & $83 \%$ & $91 \%$ & $83 \%$ & $78 \%$ & $65 \%$ \\
\hline
\end{tabular}

Tabla 5. Las emisoras en Twitter

Fuente: Elaboración propia.

\begin{tabular}{|c|c|c|c|c|c|}
\hline & Caracol Radio & W Radio & RCN Básica & La FM & Blu Radio \\
\hline $\begin{array}{l}\text { \% aumento de } \\
\text { seguidores } \\
\text { durante estudio }\end{array}$ & $3 \%$ & $7 \%$ & $3 \%$ & $9 \%$ & $10 \%$ \\
\hline $\begin{array}{l}\text { Seguidores que } \\
\text { interactúan } \\
\text { constantemente } \\
\text { con el perfil }\end{array}$ & 86.501 & 14.708 & 10.688 & 50.885 & 171.389 \\
\hline $\begin{array}{l}\text { \% de usuarios } \\
\text { comprometidos } \\
\text { con el perfil }\end{array}$ & $15 \%$ & $7 \%$ & $3 \%$ & $8 \%$ & $21 \%$ \\
\hline $\begin{array}{l}\text { Número de post } \\
\text { analizados }\end{array}$ & 621 & 799 & 139 & 548 & 553 \\
\hline $\begin{array}{l}\text { Número de } \\
\text { "Me gusta" que } \\
\text { alcanzan los } \\
\text { posts }\end{array}$ & 60.074 & 9.525 & 2.941 & 29.616 & 132.854 \\
\hline $\begin{array}{l}\text { Número de } \\
\text { comentarios } \\
\text { que generan los } \\
\text { posts }\end{array}$ & 10.240 & 3.972 & 905 & 4.489 & 21.256 \\
\hline $\begin{array}{l}\text { Número de } \\
\text { veces que son } \\
\text { compartidos los } \\
\text { posts }\end{array}$ & 18.453 & 2.329 & 358 & 4.892 & 35.315 \\
\hline $\begin{array}{l}\% \text { de post con } \\
\text { hipervínculos }\end{array}$ & $100 \%$ & $100 \%$ & $100 \%$ & $100 \%$ & $100 \%$ \\
\hline $\begin{array}{l}\% \text { de post } \\
\text { compartiendo } \\
\text { videos }\end{array}$ & $3 \%$ & $1 \%$ & $0 \%$ & $7 \%$ & $6 \%$ \\
\hline
\end{tabular}

Tabla 6. Las emisoras en Facebook

Fuente: Elaboración propia. 
la actualidad noticiosa y la agenda informativa colombiana que proponen las emisoras. En este espacio digital, se ha detectado una estrategia común basada en el establecimiento de hipervinculación entre el medio convencional, laweb-r@dioy las redes sociales, llevando a los usuarios a navegar y explorar la propuesta digital de la emisora al tiempo que acceden al sonido del medio en tiempo real o diferencial a través de Internet. Del mismo modo, también se denota la falta de interacción de la industria radiofónica en la conversación que ella misma inicia y de la que desaparece súbitamente, dejando a los usuarios sin otros interlocutores que ellos mismos.

La estrategia digital de la industria radiofónica enfoca sus acciones en evidenciar la multiplicidad de escenarios desde los que se captan y distribuyen los contenidos ofrecidos por las emisoras, los que están centrados especialmente en la agenda informativa. Ahora bien, sorprende, por una parte, que exista una baja referenciación a los videos que circulan en la red social y, por otra, el nulo reconocimiento a lo realizado por otros usuarios. En este punto, debe destacarse que el crecimiento de seguidores detectado en el periodo de estudio es significativamente bajo.

\section{INTERCONEXIÓN ANTENA-ECOSISTEMA DIGITAL}

La radio generalista, objeto de estudio, comienza a evidenciar un solapamiento entre la emisión en antena (100 temas en promedio por emisión) y su presencia digital (web-r@dio, redes sociales). Se comprueba un afán por llevar los contenidos del medio convencional a la red o, por lo menos, por promocionarlos para incrementar su consumo. Sin embargo, no sucede igual con el material de la red hacia las emisiones por onda hertziana, cuya incidencia es inferior.

Es claro que la industria se encuentra en medio de un contexto digital en el que el offline se alía con las plataformas digitales como amplificadoras de su servicio. En este punto, las páginas web y las redes sociales desempeñan un papel como instrumento narrativo de las informaciones difundidas por la antena convencional. El principal motivo de las referencias tiene que ver con las noticias del momento, la autopromoción del medio y su presencia digital, además de los comentarios que realizan personajes de la vida pública nacional. Sorprende que no se exploten los videos de sus canales aliados, así como los audios de las entrevistas realizadas por las propias emisoras (ya ubicadas en los portales web). Consecuentemente, se evidencia que los operadores apenas están iniciando el proceso digital, al no estar definidas ni consolidadas las estrategias en este campo.
El análisis de datos permitió detectar que Blu Radio, una emisora que nació bajo las dinámicas del entorno digital, es la que genera un mayor volumen de circulación entre el offline y el online, explotando temas y estableciendo un juego interacción con el público en el desarrollo de sus programas. Justamente la ausencia de diálogo con los usuarios en RCN Radio minimiza el efecto de réplica e impacto de sus publicaciones, una circunstancia que contrasta con La FM que, si bien genera un fuerte contenido digital en la web, no lo transfiere a la antena. En un punto intermedio se encuentra Caracol Básica. Llama la atención W Radio que, siendo una emisora con cobertura mundial, es la que menos se prodiga en el entorno digital, apostando por el contacto a través del correo electrónico.

La industria radiofónica colombiana, en general, se esfuerza por captar nuevos públicos bajo una estrategia digital de la difusión y gestión de sus contenidos. Esa simbiosis, que se trasluce de la relación offline y online, está centrada en cuatro ejes fundamentales: noticia (35\%), comentario (17\%), autopromoción (30\%) y tendencias (8\%), siendo el 10\% restante para entrevistas, fotos, videos, audios, créditos a un usuario y preguntas. De estas tácticas, se desprende la intensidad de la visualización del contenido, la búsqueda de consolidación de la marca ahora en el entorno digital, y el convertirse en tendencia de conversación en las redes sociales.

En la sociedad actual, las emisoras de onda hertziana no funcionan de manera aislada, sino que conforman un sistema único de comunicaciones en forma armónica y sincrónica con su propuesta digital, ya que se están dirigiendo al mismo auditorio. De hecho, la industria radiofónica colombiana está adaptando sus contenidos a un modelo comunicativo propio del entorno digital, de manera que las referencias que se hacen en la red parten de la antena (40\%), así como en sentido contrario (60\%).

El análisis del material sonoro constata que $80 \%$ de las referencias en antena a la red son cuñas grabadas que, por una parte, recuerdan al usuario la presencia digital de la emisora en la red y, por otra, informan de los perfiles en las redes sociales sin dejar de lado la invitación a buscar en laweb-r@dio algún tema en particular. Por lo general, las menciones desde la red a la antena tienen que ver con acotaciones a alguna información, con interacciones que ella misma origina o con los hashtags que impactan la actividad en las redes sociales.

\section{LA INDUSTRIA RADIOFÓNICA DE LAS WEB-R@DIO}

La industria radiofónica colombiana, a lo largo de su historia, ha afrontado diversos procesos de transición 
en los que se ha reinventado y acomodado a las situaciones propias de cada época e, incluso, a los retos tecnológicos que le son impuestos en cada momento. Actualmente, hace frente a la incorporación del medio al ecosistema digital y el estar presente en los dispositivos móviles. En ese sentido, se observa que las emisoras analizadas proyectan una arquitectura muy similar en sus portales, aunque presentan divergencias en su articulación, orientación y actuación frente a la propuesta comunicativa.

En el escenario de la convergencia, el reto para la industria radiofónica colombiana es experimentar otras formas de informar y comunicar. En este espacio, el modo en que se personaliza el gusto y se diversifican alternativas dibuja una forma de concebir el medio y desarrollar su narrativa. De hecho, la radio ya no solo depende del sonido, sino que debe manejar elementos y códigos que utiliza el lenguaje audiovisual para expresar significados coherentes y comprensibles que den sentido al relato que se ofrece:

Lo que nosotros tenemos en la red es el desarrollo de un producto, o sea nosotros, digamos Caracol S.A., está configurando un área digital que apoye lo que pasa en la cabina de radio, pero que a su vez nos enseñe cómo trasladar el formato hablado y musical a las necesidades de una audiencia en la web (Director de departamento - Caracol Radio).

La estrategia comunicativa de los portales web de la industria radiofónica colombiana está articulada por la agenda informativa nacional e internacional, el contenido específico aportado por cada programa y los podcasts destacados del día. Ello se conjuga con el streaming de la señal en antena, los blogs de los principales agentes de la emisora y el perfil corporativo de la estación y del grupo al que pertenece (incluidos los vínculos a los otros portales, web-medios). Las rutinas productivas ya no solo se concentran en el producto de antena, sino que se solapan con la convergencia de productos digitales en la red:

(...) en las diferentes reuniones en las que he estado, en las que participan las diferentes áreas convencionales y digitales del grupo, se ha dado una instrucción muy clara de una mayor integración y convergencia de contenidos. Participación activa y real, de quienes estamos al aire en el dial con lo que se hace y sucede en los diferentes medios web que conforman el conglomerado propuesto por RCN (Director de programa - RCN Radio).

La arquitectura propuesta refuerza la apropiación de las dinámicas del ecosistema digital, al tiempo que consolida el reconocimiento y credibilidad de la marca en antena. El objetivo es construir y consolidar un modelo que favorezca el consumo en tiempo diferencial, diferente del empleado en la onda hertziana, apostando por nuevos públicos. Sin embargo, esta operación debe ir acompañada además de contenidos atractivos acordes a las características y costumbres de consumo de los usuarios milennials para prevenir la desafección ya presente en otros mercados:

La radio ha tenido que reinventarse; en la creación de Blu Radio tuvimos muy claro que debíamos pensar no solo en antena, ello es cambiar el portafolio de productos y de servicios, (...), hacer cosas nuevas en el tema de radio, las redes sociales y todas las TIC que traigan la atención del público digital (Vicepresidente de radio - Blu Radio).

El ecosistema digital se constituye en una nueva unidad de negocio para la industria radiofónica bajo la interpretación particular del área directiva de cada cadena. A la luz de los datos, la innovación parece centrarse en fijar la marca en el entorno online y favorecer la circulación de contenidos con el objetivo de mantener y captar nuevos públicos. Sin embargo, la antena continúa siendo la plataforma que genera la mayoría, por no decir la totalidad, de los contenidos, por lo que las estrategias online implementadas por el momento complementan esta área de producción.

\section{CONCLUSIONES}

Los resultados de este estudio evidencian que la relación radio-usuario ya no está ligada únicamente al producto sonoro. El conjunto de los operadores colombianos busca fomentar la creación de comunidades con intereses particulares y hace uso de las redes sociales como vías de comunicación y expositores de su oferta. Desde el discurso de los directivos de la industria radiofónica colombiana se logra percibir que las emisoras se encuentran en un proceso de ensayo y error en el que buscan un elemento diferenciador que capte la atención de un público que tiene a su disposición un sinnúmero de propuestas similares con las cuales interactuar y satisfacer sus necesidades de información y consumo. El proceso hacia laweb-r@adio concentra su esfuerzo en la creación de nuevos contenidos fundamentados en narrativas transradiofónicas que responden a las lógicas de difusión cross-radial. Sin embargo, la antena es todavía omnipresente, en parte, porque continúa siendo el core del negocio, ya que la monetización de las operaciones online, con o sin sinergia con el offline, resulta extremadamente compleja. 
Lasweb-r@adiono son independientes de otras formas de comunicación convencional; los intereses del público son impulsados por las plataformas sociales y la radio no solo tiene la capacidad de amplificarlos, sino también de desarrollarlos, transmitirlos en distintas direcciones y reconvertirlos en tendencia. Tanto en el entorno digital como en el medio tradicional, la propuesta comunicativa está determinada por el gusto de la audiencia y por el criterio y estrategia del mass media. En las variables de estudio se constata una táctica de autopromoción de la radio en la red para ganar seguidores. La industria radiofónica colombiana traslada al ecosistema digital su status offline fundamentado en contenidos de actualidad informativa que acompaña de imágenes, memes e incluso música para atraer a los más jóvenes. Desde esta perspectiva, se busca seducirlos para realizar acciones de consumo en la web-r@dio y demás plataformas digitales en donde la emisora tiene presencia.

El modelo comunicativo de la radio colombiana con sus usuarios aún no asume plenamente el concepto bidireccional e interactivo del ecosistema digital. En el nuevo panorama mediático, las emisoras mantienen un fuerte control sobre el proceso y el resultado de la comunicación, dado que permiten acceso al material, propician y regulan la participación por parte de la audiencia, pero siempre bajo los intereses propios del medio. Por ahora, los usuarios se limitan mayoritariamente a comentarios con otros usuarios, a compartir las publicaciones más interesantes, mientras que la interacción con los periodistas y la propuesta de temas todavía aparecen como actividades de menor calado.

El panorama descrito pide investigar, detectar el entorno y el contexto para establecer nuevas teorías y esquemas de la disciplina en donde el flujo ya no está en una vía, sino que es múltiple, dada la presencia de varios emisores y receptores de los productos comunicativos. La aproximación a los diferentes mercados radiofónicos permite observar el proceso hacia la webr@dio y valorar su implementación, ya que su desarrollo está estrechamente conectado con la estructura industrial del sector. Además, en esta reconfiguración no puede obviarse que son las audiencias activas las que obligan a la radio a estar atenta a múltiples escenarios, donde se encuentran usuarios ávidos de interactuar e incluso participar.

\section{DISCUSIÓN}

En la actualidad, la industria radiofónica colombiana, todavía inmersa en la onda hertziana tradicional, afronta un inevitable proceso de convergencia, explorando el complejo escenario formado por los agentes de los medios, las plataformas y la audiencia en una doble vertiente: oyentes y usuarios. Así, se adoptan diferentes maneras de llegar al público: en vivo y en directo, en diferido (a la carta y podcast), mediante la exploración de enlaces, la selección de fragmentos sonoros, entre otros. Provenientes de la emisión convencional, los contenidos son integrados y explotados en la web-r@dio y app-r@dio, ciberespacios desde los que el oyenteusuario accede y consume de forma atemporal o, según sus preferencias, en simultaneidad con la emisión.

La red y el entorno digital piden a la radio escuchar, conversar y construir lo que se conoce como softpower, es decir, el poder de hacer que las cosas ocurran basadas en liderazgo y la atracción de valores, cultura y conductas. Esto se construye a través de las mismas redes en las que se forjan la imagen, la reputación y la popularidad de la marca. Sin duda, se está ante un cambio de paradigma que cuestiona la verticalidad de la tradicional comunicación radiofónica, pero que también demanda establecer unas lógicas inmersivas y convergentes que combinen la concepción de medio de masas con la búsqueda de nichos específicos, circunstancia que propicia el consumo en Internet.

Desde esta perspectiva, la industria colombiana en general aboga por una estrategia de despliegue en múltiples escenarios para buscar y contactar con el usuario. Hasta cierto punto, este comportamiento resulta lógico, ya que todavía es temprano para hablar de una transformación del modelo de negocio, una metamorfosis que pivotará entre el ranking de audiencia como muestra del volumen de oyentes y la suma de los usuarios que cada uno de los nichos temáticos aglutine con base en el consumo de sus contenidos. Mientras que los directivos están ocupados en las cuentas de resultados, los programadores buscan diseñar estrategias que reflejen el paso de la radio convencional a la webr@dio. Sin embargo, los datos muestran la construcción de dos tipos de discursos, el de la antena y el de las redes sociales, que apenas confluyen. Tan solo Blu Radio parece desmarcarse de las directrices adoptadas mayoritariamente por la industria, quizás como consecuencia de su juventud.

En la actual relación medio-usuario, el poder es cada vez más difuso. Así, la industria radiofónica colombiana entiende que las redes son el espacio para la interacción, pero su planteamiento estratégico evidencia un interés por la autopromoción y un no reconocimiento al rol de los usuarios en el entorno digital. Aunque se habla de 
dar relevancia al mensaje, en realidad lo más importante todavía es su propagación. Gracias a ella puede implicarse al usuario, que a su vez compartirá con sus contactos. Ante este panorama, a la radio colombiana se le impone un arduo trabajo: replantearse sus objetivos en aras de medir la efectividad de sus estrategias con patrones que fijen su atención no solo en valores cuantitativos sino también cualitativos, evidenciado la aparición de nichos especializados. Todos estos elementos propician ajustes y cambios acordes a las necesidades de esa comunidad de usuarios que busca satisfacer sus necesidades en la era de la web-r@adio.

En el proceso de reconfiguración, cada uno busca su lugar, en el que no solo deber ser conocido sino también recordado como una alternativa de acceso y consumo de contenidos a través de múltiples plataformas. Lo antes descrito implica, además de una convergencia, la instauración de una distribución cross-media que tenga en cuenta una narrativa conjunta respaldada por unas políticas bajo las cuales se defienden unos valores, ideas y criterios con los que se concibe y presenta el mundo. Queda atrás la etapa en la que el objetivo estaba en adaptarse al mercado radiofónico como medio de masas; hoy, el foco está en encontrar esa red de consumidores con intereses comunes y atraídos por una propuesta comunicativa que consideran verdaderamente interesante y que pueden llegar a compartir con emoción y entusiasmo.

NOTAS

1. Mercado mediático integrado, en cifras del Ministerio TIC, por 1578 estaciones radiofónicas: 667 radios comerciales, 285 radios de interés público y 626 radios comunitarias.

\section{REFERENCIAS}

Amoedo, A. \& Martínez-Costa, M. del P. (2016). Cadena Ser: nueva cultura organizacional para revitalizar el producto radiofónico [Cadena Ser: new organizational culture to revitalize the radio product]. In C. Sábada, J. A. García, \& M. del P. Martínez-Costa (Coords), Innovación y desarrollo de los cibermedios en España [Innovation and development of cybermedia in Spain] (pp. 247-254). Pamplona: Ediciones Universidad de Navarra S.A.

Ardèvol, E., Bertrán, M., Callén, B. \& Pérez, C. (2003). Etnografía virtualizada: la observación participante y la entrevista semiestructurada en línea [Virtualized ethnography: participant observation and semi-structured online interview]. Athenea Digital. Revista de Pensamiento e Investigación Social, (3), 72-92. https://doi.org/10.5565/rev/athenead/vln3.67

Arrojo, M. J. (2015). Los contenidos transmedia y la renovación de formatos periodísticos: la creatividad en el diseño de nuevas propuestas informativas [Transmedia content and the renewal of journalistic formats: creativity in the design of new information proposals]. Palabra Clave, 18(3), 746-787. https:// doi.org/10.5294/pacla.2015.18.3.6

Arzaluz, S. (2005). La utilización del estudio de caso en el análisis local [The use of the case study in the local analysis]. Región y sociedad, 17(32), 107-144. Retrieved from https://regionysociedad.colson.edu. mx:8086/index.php/rys/article/view/601/754

Ayala, T. (2014). Redes sociales, poder y participación ciudadana [Social networks, power and citizen participation]. Revista Austral de Ciencias Sociales, (26), 23-48. Retrieved from http://mingaonline. uach.cl/pdf/racs/n26/art02.pdf

Bardoel, J. (2007). Public service broadcasting in a multimedia environment. In N. Carpentier, P. Pruulmann, K. Nordenstreng, M. Hartmann, P. Vihalemm, B. Cammaerts, \& H. Nieminen (Eds.), Media technologies and democracy in an enlarged Europe: the intellectual work of the 2007 European media and communication doctoral summer school (pp. 41-54). Tartu: Tartu University Press.

Barrios, A. (2013). La radio en la era de la sociedad digital [Radio in the era of the digital society]. adComunica, (5), 39-54. https://doi.org/10.6035/2174-0992.2013.5.4 
Barrios, A. (2015). La radio colombiana frente al reto digital [Colombia digital radio and the challenge]. Comunicación y Medios, (31), 113-131. https://doi.org/10.5354/0719-1529.2015.34367

Barrios, A. (2016). La radio generalista colombiana ante el desafío digital: un modelo en transición [Colombian general radio in the face of the digital challenge: a model in transition]. Doctoral thesis. Barcelona: Universidad Autónoma de Barcelona. Retrieved from http://www.tesisenred.net/ handle/10803/386494

Barrios, A. \& Gutiérrez, M. (2016a). Transición y adaptación de la industria radiofónica colombiana privada al entorno digital desde la perspectiva de los operadores [Transition and adaptation of the Colombian private radio industry to the digital environment from the perspective of the operators]. Revista Ícono 14, 14(2), 231-255. https://doi.org/10.7195/ril4.v14i2.959

Barrios, A. \& Gutiérrez, M. (2016b). Migración de la estrategia radiofónica colombiana: del sonido a las pantallas sociales [Migration of the Colombian radio strategy: from sound to social screens]. Revista Latina de Comunicación Social, (71), 1243-1260. https://doi.org/10.4185/RLCS-2016-1144

Barrios, A. \& Zambrano, W. R. (2015). Convergencia Digital: Nuevos perfiles profesionales del periodista [Digital Convergence: New professional profiles of the journalist]. Anagramas, 13(26), 221-240. https://doi.org/10.22395/angr.v13n26al1

Batista, J. M. (2004). La empresa informativa, una asignatura pendiente: ¿cómo definir las estrategias de gestión de personal? [The information company, a pending issue: how to define personnel management strategies?]. Revista Latina de Comunicación Social, 7(58), 1-6. Retrieved from http://www.ull.es/publicaciones/latina/20045828bacallado.pdf

Bernal, C. A. (2006). Metodología de la Investigación para Administración, Economía, Humanidades y Ciencias Sociales [Research Methodology for Administration, Economics, Humanities and Social Sciences]. Mexico City: Pearson.

Bonini, T. \& Monclús, B. (2015). Radio Audiences and participation in the Age of Network Society. New York: Routledge.

Campos-Freire, F. (2015). Adaptación de los medios tradicionales a la innovación de los metamedios [Adaptation of traditional media to the metamedia innovation]. El profesional de la información, 24(4), 441-450. https://doi.org/10.3145/epi.2015.jul.11

Cebrián, M. (2009). Comunicación interactiva en los cibermedios [Interactive communication in the cybermedia]. Comunicar, 17(33), 15-24. https://doi.org/10.3916/c33-2009-02-001

Centro Nacional de Consultoría (2016). Estudio continuo de audiencia radial ECAR, tercera ola 2016 [Continuous study of radial audience ECAR third wave 2016]. Bogota: Colombia.

Cerezo, P. (2016). La generación Z y la información [Generation Z and information]. Revista estudios de la juventud, (114), 95-109. Retrieved from http://www.injuve.es/sites/default/files/2017/28/ publicaciones/documentos_7._la_generacion_z_y_la_informacion.pdf

Colussi, J. (2013). Propuesta metodológica para el análisis de blogs periodísticos [Methodological proposal for the analysis of journalistic blogs]. Intercom-Revista Brasileira de Ciências da Comunicação, 36(2), 197-218. Retrieved from http://portcom.intercom.org.br/revistas/index.php/revistaintercom/ article/view/1789

Domingo, D., Salaverría, R., Aguado, J. M., Cabrera, M. de los Á., Edo, C., Masip, P., ... \& Portilla, I. (2007). Four Dimensions of Journalistic Convergence: A preliminary approach to current media trends at Spain. Paper presented at the 8th International Symposium on Online Journalism. Austin, Texas.

Fernández, B. (2014). La radio ante el desafío de las nuevas audiencias [The radio to the challenge of the new audiences]. Razón y Palabra, 18(87). Retrieved from http://www.razonypalabra.org.mx/N/N87/ V87/01_Fernandez_V87.pdf

García-Alonso Montoya, P. (2014). La empresa informativa busca nuevos modelos de negocio [Media are looking for new business model]. Historia y Comunicación Social, 19, 729-741. https://doi.org/10.5209/ rev_HICS.2014.v19.45173 
García-de-Torres, E. (2010). Contenido generado por el usuario: aproximación al estado de la cuestión [User generated content: a state of the situation]. El profesional de la información, 19(6), 585-594. https://doi.org/10.3145/epi.2010.nov.04

González, M. J. \& Salgado, C. (2011) Perspectivas sobre la comunicación radiofónica dentro de su evolución mediática. La figura del comunicador de información [Perspectives on radio communication within its media evolution. The figure of the information Communicator]. In M.A. Ortiz \& N. López (Eds), Radio 3.0 Una nueva radio para una nueva era: la democratización de los contenidos [Radio 3.0 A new radio for a new era: the democratization of content] (pp. 165-188) Madrid: Editorial Fragua.

Gutiérrez, M., Monclús, B., \& Martí, J. (2014). Radio y jóvenes, una encrucijada de intereses y expectativas [Radio and young people, a crossroads of interests and expectations]. In A. Huertas Bailén \& M. Figueras Maz (Eds.), Audiencias juveniles y cultura digital [Youth audiences and digital culture] (pp. 107-123). Bellaterra: Institut de la Comunicació, Universitat Autònoma de Barcelona.

Huertas, A. \& Figueras M. (Eds.) (2014). Audiencias juveniles y cultura digital [Youth audiences and digital culture]. Bellaterra: Institut de la Comunicaciò, Universitat Autònoma de Barcelona.

López, G. (Ed.) (2005). El ecosistema digital: Modelos de comunicación, nuevos medios y público en Internet [The digital ecosystem: Communication models, new media and public on the Internet]. Valencia: Servei de Publicacions de la Universitat de València.

López, N. \& Gómez, L. (2014). Claves de la desafección juvenil hacia la radio generalista española [Keys to youth disaffection towards Spanish general radio]. Actas - VI Congreso Internacional Latina de Comunicación Social. Spain: Universidad de La Laguna. Retrieved from http://www.revistalatinacs. org/14SLCS/2014_actas/022_Lopez.pdf

López, B. \& Martínez, G. (2014). Comunicación 360 [360 communication]. In T. Pintado \& J. Sánchez (Coords.), Nuevas tendencias en comunicación estratégica [New trends in strategic communication] (pp. 19-48). Madrid: ESIC Editorial.

Martí, J. (2011). Propuesta de una herramienta de análisis de contenido para el emplazamiento de producto en contenidos audiovisuales [A Proposal of a Tool for Content Analysis of Product Placement in Audiovisual Content]. Pensar la Publicidad, 5(2), 65-92. https://doi.org/10.5209/rev_ PEPU.2011.v5.n2.37864

Martínez, P. C. (2006). El método de estudio de caso: estrategia metodológica de la investigación científica [The method of case study: methodological strategy of scientific research]. Pensamiento $\mathcal{E}$ Gestión, (20), 165-193. Retrieved from http://rcientificas.uninorte.edu.co/index.php/pensamiento/ article/viewFile/3576/2301

Martínez-Costa, M. del P. (2015). Radio y nuevas narrativas: de la crossradio a la transradio [Radio and new narratives: from the crossradio to the transradio]. In M. Oliveira \& F. Ribeiro (Eds.) Radio, sound and Internet. Proceedings of Net Station International Conference (pp. 168-187). Brasil: Centro de Estudos de Comunicação e Sociedade (CECS), Universidade do Minho.

Meso, K., Larrondo, A., Peña, S., \& Rivero, D. (2014). Audiencias activas en el ecosistema móvil. Análisis de las opciones de interacción de los usuarios en los cibermedios españoles a través de la web, los teléfonos móviles y las tabletas [Active audiences in the mobile ecosystem: Analysis of the interaction options in Spanish cybermedia through websites, mobile telephones and tablets]. Hipertext.net: Anuario Académico sobre Documentación Digital y Comunicación Interactiva, (12). https://doi.org/10.2436/20.8050.01.9

Ortiz, M. A. \& López, N. (2011). Radio 3.0 Una nueva radio para una nueva era. La democratización de los contenidos [Radio 3.0 A new radio for a new era. The democratization of content]. Madrid: Fragua

Pavlik, J. V. (2004). A Sea-Change in Journalism: Convergence, Journalists, their Audiences and Sources. Convergence, 10(4), 21-29. https://doi.org/10.1177/135485650401000404

Peñafiel, C. (2016). Reinvención del periodismo en el ecosistema digital y narrativas transmedia [Reinventing journalism in the digital ecosystem and transmedia story-telling]. adComunica, (12), 163-182. https://doi.org/10.6035/2174-0992.2016.12.10 
Perona, J. J., Barbeito, M. L., \& Fajula, A. (2014). Los jóvenes ante la sono-esfera digital: medios, dispositivos y hábitos de consumo sonoro [Young people in the digital sonosphere: media digital, media devices and audio consumption habits]. Communication \& Society, 27(1), 205-224. Retrieved from https://www.unav.es/fcom/communication-society/es/articulo.php?art_id=487

Piñero-Otero, T. \& Videla, J. (2013). La participación de los oyentes en las radios APP españolas. Prácticas convencionales en la era de la portabilidad [The participation of the audience in the Spanish radio apps. Conventional practices in the era of portability]. adComunica, (5), 67-89. https://doi.org/10.6035/2174-0992.2013.5.6

Preciado, A. \& Monsalve, M. F. (2008). El liderazgo en la gestión de la empresa informativa [The leadership in the management of the informative Company]. Revista Ciencias Estratégicas, 16(19), 79-96. Retrieved from https://revistas.upb.edu.co/index.php/cienciasestrategicas/article/view/596/534

Ramos del Cano, F. (2014). Redes sociales y participación radiofónica: Análisis de caso de Twitter y Facebook en la Cadena SER [Social networks and radio participation: Case analysis of Twitter and Facebook in the Cadena SER]. Ambitos: revista internacional de comunicación, (25), 66-76. Retrieved from http://institucional.us.es/ambitos/?p=1105

Ribes, X., Monclús, B., Gutiérrez-García, M., \& Martí, J. M. (2017). Aplicaciones móviles radiofónicas: adaptando las especificidades de los dispositivos avanzados a la distribución de los contenidos sonoros [Mobile radio applications: adapting the specificities of advanced devices to the distribution of sound contents]. Revista de la Asociación Española de Investigación de la Comunicación, 4(7), 29-39. Retrieved from http://www.revistaeic.eu/index.php/raeic/article/view/102

Rivadeneyra, C. (2008). Pugna, convergencia y diversidad de la radio latinoamericana en el escenario digital [Pugna, convergence and diversity of Latin American radio in the digital scenario]. In L. Ortega Carmona (Comp.), Memorias de la $7^{\circ}$ Bienal Internacional de Radio México [Memories of the 7th International Radio Mexico Bienniall. Colonia del Valle: Radio Educación.

Salaverría, R. (2010). Estructura de la convergencia [Structure of convergence]. In X. López \& X. Pereira (Eds.), Convergencia digital. Reconfiguración de los medios de comunicación en España [Digital convergence. Reconfiguration of the media in Spain] (pp. 27-40). Santiago de Compostela: Servicio Editorial de la Universidad de Santiago de Compostela.

Salaverría, R. \& García, A. (2008). La convergencia tecnológica en los medios de comunicación: retos para el periodismo [Technological convergence in the media: challenges for journalism]. Tripodos, (23), 31-47. Retrieved from http://www.raco.cat/index.php/Tripodos/article/view/118910/154114

Scolari, C. A. (2013). Narrativas Transmedia [Transmedia Narratives]. Madrid, Spain: Editorial Grupo Planeta.

Soengas, X. (2013). Retos de la radio en los escenarios de la convergencia digital [Radio challenges in digital convergence sceneries]. adComunica, (5), 23-36. https://doi.org/10.6035/2174-0992.2013.5.3

Sosa, G. (2000). Radio e Internet: la inevitable convergencia tecnológica [Radio and Internet: the inevitable technological convergence]. In O. Islas (Coord.), Internet: el medio inteligente [Internet: the intelligent medium] (pp. 112-123). Mexico City: Editorial Patria.

Stake, R. E. (1998). Investigación con estudio de casos [Research with case studies]. Madrid, Spain: Ediciones Morata.

Yacuzzi, E. (2005). El estudio de caso como metodología de investigación: Teoría, mecanismos causales, validación [The case study as a research methodology: Theory, causal mechanisms, validation] $\left(\mathrm{N}^{\circ}\right.$ 296). Serie Documentos de Trabajo, Universidad del CEMA: Area: negocios.

Yuste, B. (2015). Las nuevas formas de consumir información de los jóvenes [New ways of consuming information from young people]. Revista Estudios de Juventud, (108), 179-191. Retrieved from http://www.injuve.es/sites/default/files/14.\%20Las\%20nuevas\%20forma\%20de\%20consumir\%20 informaci\%C3\%B3n\%20de\%20los\%20j\%C3\%B3venes.pdf 


\section{SOBRE LOS AUTORES}

Andrés Barrios Rubio, profesor titular del Departamento de Comunicación Social y Cinematografía de la Universidad de Bogotá Jorge Tadeo Lozano, del cual es director desde agosto de 2017. Desarrolla su actividad investigadora en el uso de las redes sociales como espacio para la interculturalidad, el impacto de las TIC en la formación de los comunicadores y en el análisis de las sinergias entre el on air y el online en la industria radiofónica colombiana, temas sobre los que versan sus publicaciones académicas.

Maria Gutiérrez García, profesora titular del Departamento de Comunicación Audiovisual y Publicidad de la Universitat Autònoma de Barcelona (UAB), del cual es directora desde septiembre de 2015. Es miembro fundadora del equipo de investigación de l'Observatori de la Ràdio a Catalunya (GRISS-UAB), donde participa activamente en la codirección y realización de proyectos de investigación. Sus publicaciones están relacionadas con los contenidos radiofónicos en el entorno digital, el uso de las redes sociales, la audiencia juvenil y los nuevos modelos de negocio. 